\title{
Travel-associated Legionnaires' disease (TALD) cluster definition
}

Maria Cristina Rota, A. Bella, M. G. Caporali, A. Nicolau, V. Drasar, M. L. Ricci, M. Scaturro, M. Gumá, S. Crespi

\section{Source}

M. C. Rota, A. Bella, M. G. Caporali, A. Nicolau, V. Drasar, M. L. Ricci. (2018). Travelassociated Legionnaires' disease: would changing cluster definition lead to the prevention of a larger number of cases? Epidemiol. Infect., vol. 147

Sites associated with at least two cases regardless of the time elapsed between them 